Tecnociencia, Vol. 23, $\mathrm{N}^{\circ} 1: 26-49$

Enero-junio 2021

\title{
COMPARACIÓN DE LAS PRINCIPALES MOSCAS NECRÓFAGAS ATRAÍDAS POR HÍGADOS HUMANOS EN ESTADO DE DESCOMPOSICIÓN, EXPUESTOS A DIFERENTES INTERVALOS DE TIEMPO, EN UN ÁREA URBANA DE LA PROVINCIA DE PANAMÁ.
}

\section{Garcés, P.A (iD 1., M.B. Rosa ${ }^{2}$, O.J. Portillo² I.I. Ross ${ }^{2}$, C.O. Jiménez $^{2}$, C.M. Moreno' ${ }^{2}$ J. J. Cobos' ${ }^{2}$, O. Zapata ${ }^{2}$, C. Chiari², V. $\mathrm{Ku}^{2}$, V. Gutiérrez. Medina², Y. Góndola², C. Mendieta, C. Chiari, I. Ochoa y W. Pitti.}

${ }^{1}$ Universidad de Panamá/FCINET, Vicerrectoría de Investigación y Postgrado, Programa Centroamericano de Maestría en Entomología, Depto. de Zoología ${ }^{2}$ Ministerio Público, Instituto de Medicina legal y ciencias forenses.

E-mail: 1perchysg@gmail; ${ }^{2}$ mayita2310@hotmail.com, ${ }^{2}$ mdomarportillo@gmail.com, , 2iiross2@gmail.com, , 2drmoreno1302@gmail.com, 2jessica.j.cobos@gmail.com, 2oazt07@hotmail.com,_2celinachiari@gmail.com,_2valetakp@gmail.com, 2orquidealorim@gmail.com,_2dragutierrez07@hotmail.com, 2mdcmendieta@hotmail.com, , 22ygondola25@yahoo.es, 22ochoaisisp@gmail.com ${ }^{2}$ pitti.wilfredo@gmail.com

\section{RESUMEN}

Se obtuvieron cuatro muestras de hígados humanos, de aproximadamente de 150 a 200 g. de la Morgue Judicial de Panamá. Los tejidos se expusieron a intervalos de tiempo que iban desde las primeras 12 horas, incluyendo las $24,48,72$ y hasta 96 horas de descomposición. Las capturas de las moscas se realizaron durante tres horas continuas.

En total se capturaron 1003 ejemplares, distribuidos en tres familias y 11 especies: 
Calliphoridae, Sarcophagidae y Muscida. La familia que mayormente atrapada en los tejidos fue la Calliphoridae seguida de la Sarcophagidae. Las especies más abundantes en el área correspondió a Chrysomya megacephala, con 464 ejemplares y Lucilia cuprina con 117 ejemplares, seguido de Lucilia sericata con 55 ejemplares y Lucilia eximia con 47 ejemplares. El objetivo de esta investigación fue conocer las principales especies de moscas necrófagas que arriban a los hígados humanos, expuestos a diferentes intervalos, en un área urbana de la provincia de Panamá.

PALABRAS CLAVES: hígados humanos, intervalos de tiempo, Chrysomya megacephala, Lucilia cuprina, Lucilia sericata.

\title{
COMPARISON OF THE MAIN NECROPHAGUS FLIES ATTRACTED BY HUMAN LIVES IN A STATE OF DECOMPOSITION, EXPOSED TO DIFFERENT TIME INTERVALS, IN AN URBAN AREA OF THE PROVINCE OF PANAMA.
}

\begin{abstract}
Four samples of human's livers of approximately $150-200 \mathrm{~g}$. were obtained from the Judicial Mortuary of Panama. The livers were used at different interval from 12, 24, 48, 72 and 96 hours of decomposition. The flies were captured during 3 hours with intervals of 10, 15 and $20 \mathrm{~min}$, until the 3 hours sampling was completed. A total amount of 1003 flies were captured, distributed in 3 families: Calliphoridae, Sarcophagide y Muscidae. To capture 11 species, the more abundant species corresponded to Chrysomya megacephala, with 464 specimens, Lucila cuprina, with 117 specimens, Lucilia sericata, with 55 specimens and Lucilia eximia, with 47 specimens. The purpose of this research was knew the colonization of human liver, exposed at different time intervals in an urban area of the province of Panama.
\end{abstract}

KEY WORDS: human livers, time intervals, Chrysomya megacephala, Tecnociencia, Vol. 23, $N^{\circ} 1$ 
Lucilia cuprina, Lucilia sericata.

\section{INTRODUCCIÓN}

La Entomología Forense se basa en el estudio de Artrópodos involucrados en eventos tales como suicidio, asesinato, muertes naturales, abusos físicos y tráficos de contrabando (Catts y Goff, 1992). $\mathrm{Su}$ utilidad se basada en el hecho de que los artrópodos colonizan los cadáveres frescos, utilizándolos como recurso alimenticio en forma secuencial y predecible haciendo de la descomposición cadavérica un proceso medible (Byrd y Castner, 2001). Existen dos métodos que se pueden usar de forma conjunta para determinar la fecha de la muerte. En el primero, la estimación se basa en el estudio del crecimiento larval de una o dos especies de insectos y el segundo, utiliza la composición de la comunidad de artrópodos encontradas en el cadáver y se compara con los patrones conocidos de sucesión de la fauna local, para hábitat y condiciones ambientales similares (Camacho, 2003).

Diversos autores en diferentes países han realizados estudios para conocer los insectos asociados a la descomposición cadavérica. Muchos de ellos se han realizados tanto áreas urbanas como en áreas boscosas. Algunos de los realizados en áreas urbanas son los de (Centeno., Almorza y Arnillas, 2004; Carvalho, Thyssen, Goff y Linhares, 2004; Barbosa, Mello-Patiu., Ururahy-Rodrigues, Guimarães Barbosa y Carvalho Queiroz, 2010; Amat, Ramíre-Mora, Buenaventura y Gómepinerez, 2013; Ramos-Pastrana, Velasquez-Valencia y Wolff, 2014). Empleando diferentes biomodelos no humanos como sustratos entre ellos (pollos, gatos, perros, ratas, conejos), debido a las objeciones éticas y morales en cuanto al uso de cadáveres humanos para este fin (Smith, 
1986). El cerdo doméstico Sus scrofa L. ha sido el biomodelo más utilizado, debido a las ventajas que ofrece entre estas, su gran parecido con la descomposición del ser humano (Catts y Goff, 1992), las semejanzas en su anatomía interna, la distribución de grasa corporal, el tamaño de la cavidad del torácica y la escasez de pelo hacen de este animal un biomodelo adecuado para este tipo de estudios (Sanabria, 2008; Schoenly, Hankell, Mills, Bieme, Larsen y Lee, 2006).

El objetivo de este estudio fue conocer a las principales especies de moscas que arriban a los diferentes hígados humanos, expuestos a diferentes intervalos de tiempo, en un área urbana de la provincia de Panamá

\section{MATERIALES Y MÉTODOS}

Se obtuvieron cuatro muestras de hígados humano, de distintos cadáveres, de aproximadamente de 150 a 200 g en la Morgue Judicial de Panamá. Estos hígados fueron extraídos de los cadáveres conservados en la Morgue, con autorización de la coordinadora de la Morgue Judicial de Panamá y con el visto bueno del Director General. Los tejidos más avanzados se obtuvieron, primeramente, dejándolos descomponer, hasta que tuvieran el tiempo necesario para el estudio. Mientras que los tejidos más frescos se obtuvieron a las 12 horas, es decir de cadáveres más recientes, que se encontraran en el tiempo requerido.

Los trozos de hígados fueron cortados con bisturí y pesados para que fueran homogéneos. Se emplearon hígados con intervalos de 12 horas, 
$24,48,72$, y 96 horas de descomposición y se colocaron en envases plásticos, mantenidos a temperatura ambiente, siguiendo la metodología de (Garcés y Rosas, 2016). Posteriormente, los diversos hígados fueron trasladados al sitio de estudio, próximo a una quebrada, cerca de árboles frutales, en un área urbana, de los Llanos de Curundú, en Albrook, entre las coordenadas geográficas $8^{\circ} 58^{\prime} 55.78^{\prime \prime} \mathrm{N}$ y $79^{\circ}$ 33'04.36" O.

Los dos primeros hígados se emplearon en los dos primeros intervalos de tiempo 12 y 24 horas, estaban en estado cromático o frescos y los dos últimos de 72 y 96 horas, estaban en el estado enfisematoso o de pudrición avanzada.

Los insectos fueron capturados durante tres horas continuas, cada hora fue subdividida en intervalos para conocer el momento preciso en que arriban las moscas a los hígados. La primera hora, se subdivido en intervalos de 10 minutos hasta completar la primera hora de muestreo. Se consideró como arribo a los especímenes que se posaron, próxima o sobre los hígados.

La segunda hora de muestreada, fue subdividida en intervalos de 15 minutos, hasta completar la segunda hora y, la tercera hora se dividió en intervalos de 20 minutos hasta completar la tercera hora de muestreo. Los insectos capturados fueron colocados en viales debidamente rotulados que contenían alcohol al 70\%. También se colectaron insectos sobre la vegetación circundante con la ayuda de una red entomológica. Se fotografiaron las actividades que los insectos realizaban sobre la vegetación y sobre los hígados para documentar la actividad. Los especímenes capturados fueron trasladados al Programa 
Centroamericano de Maestría en Entomología, de la Universidad de Panamá, donde se procedió a separarlos y montarlos en alfileres entomológicos. La identificación de los mismos se realizó mediante el empleo de un estereoscopio y, con ayuda de las claves taxonómicas de Amat y Wolff, (2008).

\section{RESULTADOS}

En total se capturaron 1003 ejemplares, distribuidos en tres familias y en 11 especies. Las tres familias fueron Calliphoridae, Sarcophagidae y Mucidae. Las especies más abundantes fueron a Chrysomya megacephala, con 464 especímenes, seguido de Lucilia cuprina, con 117 especímenes, Lucilia sericata, con 55 especímenes y Lucilia eximia, con 47 especímenes. Las especies más abundantes de las Sarcophagidae fueron las morfoespecies $\mathrm{Sp} 2$, con 37 especímenes, seguido de la $\mathrm{Sp} 1$, con ocho especímenes y la $\mathrm{Sp} 3$ con cuatro especímenes.

En el hígado con 12 horas de exposición, se capturó un total de 31 ejemplares, distribuidos en dos familias (Calliphoridae y Sarcophagidae) y cuatro especies. La mayor captura correspondió a la especie Lucilia sericata, con 23 especímenes, que representaron el 74\% de las capturas, seguido de la especie Compsomyiops sp con cinco ejemplares que correspondieron al $16 \%$ de las capturas (Cuadro 1). 
Cuadro 1. Especies registradas en el hígado humano expuesto a las 12 horas de haber sido extraído del cadáver.

\begin{tabular}{|c|c|c|c|c|c|c|c|c|c|c|c|c|c|c|c|c|}
\hline \multirow{2}{*}{ ESPECIES DE MOSCAS } & \multicolumn{6}{|c|}{ PRIMERA HORA (MIN) } & \multirow{2}{*}{ TOTAL } & \multicolumn{4}{|c|}{ SEGUNDA HORA (MIN) } & \multirow{2}{*}{ TOTAL } & \multicolumn{3}{|c|}{ TERCERA HORA (MIN) } & \multirow{2}{*}{ TOTAL } \\
\hline & 10 & 20 & 30 & 40 & 50 & 60 & & 15 & 30 & 45 & 60 & & 20 & 40 & 60 & \\
\hline \multicolumn{17}{|l|}{ Calliphoridae } \\
\hline Lucilia sericata & 1 & 0 & 0 & 2 & 5 & 1 & 7 & 1 & 1 & 0 & 3 & 5 & 4 & 4 & 3 & 11 \\
\hline Lucilia cuprina & 0 & 0 & 0 & 0 & 0 & 0 & 0 & 0 & 0 & 0 & 0 & 0 & 0 & 0 & 0 & 0 \\
\hline Chrysomya rufifacies & 0 & 0 & 0 & 0 & 0 & 0 & 0 & 0 & 0 & 0 & 0 & 0 & 0 & 0 & 0 & 0 \\
\hline Chrysomya megacephala & 0 & 0 & 0 & 0 & 0 & 0 & 0 & 0 & 0 & 0 & 0 & 0 & 0 & 0 & 0 & \\
\hline Compsomyiops $s p$ & 0 & 1 & 0 & 4 & 0 & 0 & 5 & 0 & 0 & 0 & 0 & 0 & 0 & 0 & 0 & 0 \\
\hline \multicolumn{17}{|l|}{ Sarcophagidae } \\
\hline SP1 & 1 & 0 & 0 & 0 & 0 & 0 & 1 & 0 & 0 & 0 & 0 & 0 & 0 & 0 & 0 & 1 \\
\hline Sp 2 & 0 & 0 & 0 & 0 & 0 & 0 & 0 & 0 & 0 & 1 & 0 & 0 & 0 & 0 & 0 & 1 \\
\hline Sp3 & 0 & 0 & 0 & 0 & 0 & 0 & 0 & 0 & 0 & 0 & 0 & 0 & 0 & 0 & 0 & 0 \\
\hline TOTALES & & & & & & & 13 & & & & & 5 & & & & 13 \\
\hline
\end{tabular}

En el hígado con 24 horas de exposición, se capturaron 35 ejemplares, distribuidos en dos familias (Calliphoridae y Sarcophagidae) y en cinco especies. La especie más capturada fue Lucilia sericata, con 21 especímenes, que correspondió a el 60\% de las capturas, seguido de la especie Compsomyiops sp, con siete individuos que representó el 20\% de las capturas (Cuadro 2).

Cuadro 2. Especies registradas en el hígado humano expuesto a las 24 horas de haber sido extraído del cadáver.

\begin{tabular}{|c|c|c|c|c|c|c|c|c|c|c|c|c|c|c|c|c|}
\hline \multirow{2}{*}{ ESPECIES DE MOSCAS } & \multicolumn{6}{|c|}{ PRIMERA HORA (MIN) } & \multirow{2}{*}{ TOTAL } & \multicolumn{4}{|c|}{ SEGUNDA HORA (MIN) } & \multirow{2}{*}{ TOTAL } & \multicolumn{3}{|c|}{ TERCERA HORA (MIN) } & \multirow{2}{*}{ TOTAL } \\
\hline & 10 & 20 & 30 & 40 & 50 & 60 & & 15 & 30 & 45 & 60 & & 20 & 40 & 60 & \\
\hline \multicolumn{17}{|l|}{ Calliphoridae } \\
\hline Lucilia sericata & 1 & 0 & 0 & & 5 & 3 & 9 & 2 & 1 & 0 & 3 & 6 & 2 & 1 & 3 & 6 \\
\hline Lucilia cuprina & 0 & 0 & 0 & 0 & 1 & 0 & 1 & 0 & 0 & 1 & 0 & 1 & 0 & 0 & 1 & 1 \\
\hline Chrysomya rufifacies & 0 & 0 & 0 & 0 & 0 & 0 & 0 & 0 & 0 & 0 & 0 & 0 & 0 & 0 & 0 & 0 \\
\hline Chrysomya megacephala & 0 & 0 & 0 & 0 & 0 & 0 & 0 & 0 & 0 & 0 & 0 & 0 & 0 & 0 & 0 & 0 \\
\hline Compsomyiops $s p$ & 0 & 1 & 0 & 4 & 0 & 0 & 5 & 0 & 0 & 0 & 1 & 1 & 0 & 1 & 0 & 1 \\
\hline \multicolumn{17}{|l|}{ Sarcophagidae } \\
\hline SP1 & 1 & 0 & 0 & 0 & 0 & 0 & 1 & 0 & 0 & 0 & 0 & 0 & 0 & 1 & 0 & 1 \\
\hline Sp 2 & 0 & 0 & 0 & 0 & 0 & 0 & 0 & 0 & 0 & 1 & 0 & 1 & 0 & 1 & 0 & 1 \\
\hline Sp3 & 0 & 0 & 0 & 0 & 0 & 0 & 0 & 0 & 0 & 0 & 0 & 0 & 0 & 0 & 0 & 0 \\
\hline TOTALES & & & & & & & 16 & & & & & 9 & & & & 10 \\
\hline
\end{tabular}

En el hígado con 48 horas de exposición, se capturaron un total de 58

Garcés, P.A \& Colaboradores 
ejemplares, distribuidos en dos familias (Calliphoridae y Sarcophagidae) y en cinco especies. La especie más capturada fue Chrysomya rufifacies, con 52 especímenes, que correspondieron al 89\% de las capturas (Cuadro 3).

Cuadro 3. Especies registradas en el hígado humano expuesto a las 48 horas de haber sido extraído del cadáver.

\begin{tabular}{|c|c|c|c|c|c|c|c|c|c|c|c|c|c|c|c|c|}
\hline \multirow{2}{*}{ ESPECIES DE MOSCAS } & \multicolumn{6}{|c|}{ PRIMERA HORA (MIN) } & \multirow{2}{*}{ TOTAL } & \multicolumn{4}{|c|}{ SEGUNDA HORA (MIN) } & \multirow{2}{*}{ TOTAL } & \multicolumn{3}{|c|}{ TERCERA HORA (MIN) } & \multirow{2}{*}{ TOTAL } \\
\hline & 10 & 20 & 30 & 40 & 50 & 60 & & 15 & 30 & 45 & 60 & & 20 & 40 & 60 & \\
\hline \multicolumn{17}{|l|}{ Calliphoridae } \\
\hline Lucilia sericata & 0 & 0 & 0 & 0 & 0 & 0 & 0 & 0 & 0 & 0 & 0 & 0 & 0 & 0 & 0 & 0 \\
\hline Lucilia cuprina & 0 & 0 & 1 & 0 & 0 & 0 & 1 & 0 & 0 & 1 & 0 & 1 & 0 & 0 & 0 & 0 \\
\hline Lucilia eximia & 0 & 0 & 0 & 0 & 0 & 0 & 0 & 0 & 0 & 0 & 0 & 0 & 0 & 0 & 0 & 0 \\
\hline Chrysomya rufifacies & 1 & 2 & 3 & 3 & 2 & 2 & 13 & 6 & 7 & 3 & 6 & 22 & 3 & 4 & 8 & 17 \\
\hline Chrysomya megacephala & 0 & 0 & 0 & 0 & 0 & 0 & 0 & 0 & 0 & 0 & 0 & 0 & 0 & 0 & 0 & 0 \\
\hline \multicolumn{17}{|l|}{ Sarcophagidae } \\
\hline SP1 & 0 & 0 & 0 & 0 & 1 & 0 & 1 & 0 & 0 & 0 & 0 & 0 & 0 & 1 & 0 & 1 \\
\hline Sp2 & 0 & 0 & 0 & 0 & 0 & 0 & 0 & 0 & 0 & 0 & 0 & 0 & 0 & 0 & 0 & 0 \\
\hline Sp3 & 0 & 0 & 0 & 0 & 1 & 0 & 1 & 0 & 0 & 0 & 0 & 0 & 0 & 0 & 0 & 1 \\
\hline
\end{tabular}

En el hígado con 72 horas de exposición, se capturaron un total de 312 ejemplares, distribuidos en dos familias (Calliphoridae y Sarcophagidae) y en seis especies. La mayor captura correspondió a la especie Lucilia cuprina, con 98 especímenes lo que representa el 31\% de las capturas, seguido de la especie Chrysomya rufifacies, con 43 especímenes, que correspondió a el 14\% y, por último, a Lucilia sericata, con 10 especímenes, que representó el 30\% de las capturas (Cuadro 4). 
Cuadro 4. Especies registradas en el hígado humano expuesto a las 72 horas de haber sido extraído del cadáver.

\begin{tabular}{|c|c|c|c|c|c|c|c|c|c|c|c|c|c|c|c|c|}
\hline \multirow{2}{*}{ ESPECIES DE MOSCAS } & \multicolumn{6}{|c|}{ PRIMERA HORA (MIN) } & \multirow{2}{*}{ TOTAL } & \multicolumn{4}{|c|}{ SEGUNDA HORA (MIN) } & \multirow{2}{*}{ TOTAL } & \multicolumn{3}{|c|}{ TERCERA HORA (MIN) } & \multirow{2}{*}{ TOTAL } \\
\hline & 10 & 20 & 30 & 40 & 50 & 60 & & 15 & 30 & 45 & 60 & & 20 & 40 & 60 & \\
\hline \multicolumn{17}{|l|}{ Calliphoridae } \\
\hline Lucilia cuprina & 0 & 7 & 0 & 15 & 7 & 3 & 32 & 21 & 14 & 9 & 0 & 44 & 10 & 11 & 2 & 22 \\
\hline Lucilia sericata & 0 & 0 & 0 & 0 & 0 & 1 & 1 & 0 & 0 & 1 & 5 & 6 & 2 & 1 & 0 & 3 \\
\hline Lucilia eximia & 0 & 0 & 0 & 0 & 0 & 0 & 0 & 0 & 0 & 0 & 0 & 0 & 0 & 0 & 0 & \\
\hline Chrysomya rufifacies & 3 & 2 & 3 & 1 & 2 & 1 & 11 & 23 & 2 & 2 & 3 & 30 & 0 & 1 & 1 & 2 \\
\hline Chrysomya megacephala & 0 & 0 & 0 & 0 & 0 & 0 & 0 & 0 & 0 & 0 & 0 & 0 & 0 & 0 & 0 & 0 \\
\hline \multicolumn{17}{|l|}{ Sarcophagidae } \\
\hline SP1 & 0 & 1 & 0 & 0 & 0 & 0 & 1 & 0 & 0 & 0 & 0 & 0 & 0 & 0 & 0 & 0 \\
\hline SP2 & 0 & 0 & 0 & 1 & 0 & 0 & 0 & 0 & 0 & 0 & 1 & 0 & 0 & 0 & 0 & 0 \\
\hline SP3 & 0 & 0 & 1 & 0 & 0 & 0 & 1 & 0 & 1 & 0 & 0 & 1 & 0 & 0 & 0 & 0 \\
\hline TOTALES & 3 & 10 & 4 & 17 & 9 & 5 & 46 & 44 & 17 & 12 & 9 & 81 & 12 & 13 & 3 & 27 \\
\hline
\end{tabular}

En el hígado con 96 horas de exposición, se capturaron un total de 591 ejemplares, distribuidos en tres familias (Calliphoridae, Sarcophagidae y Muscidae) y en 11 especies. La especie más captura fue Chrysomya megacephala, con 464 especímenes, lo que representó el 78\% de las capturas, seguido de la especie Chrysomya rufifacies, con 53 especímenes, que representan el 8.9\% y, por último, la especie Lucilia eximia, con 47 especímenes que corresponden al 8\% de las capturas (Cuadro 5). 
Cuadro 5. Especies registradas en el hígado humano expuesto a las 96 horas de haber sido extraído del cadáver.

\begin{tabular}{|c|c|c|c|c|c|c|c|c|c|c|c|c|c|c|c|c|}
\hline \multirow{2}{*}{ ESPECIES DE MOSCAS } & \multicolumn{6}{|c|}{ PRIMERA HORA (MIN) } & \multirow{2}{*}{ TOTAL } & \multicolumn{4}{|c|}{ SEGUNDA HORA (MIN) } & \multirow{2}{*}{ TOTAL } & \multicolumn{3}{|c|}{ TERCERA HORA (MIN) } & \multirow{2}{*}{ TOTAL } \\
\hline & 10 & 20 & 30 & 40 & 50 & 60 & & 15 & 30 & 45 & 60 & & 20 & 40 & 60 & \\
\hline \multicolumn{17}{|l|}{ Calliphoridae } \\
\hline Lucilia sericata & 1 & 0 & 3 & 0 & 0 & 0 & 4 & 0 & 0 & 0 & 0 & 0 & 0 & 0 & 0 & 0 \\
\hline Lucilia cuprina & 2 & 0 & 2 & 1 & 1 & 1 & 7 & 0 & 1 & 1 & 1 & 2 & 1 & 2 & 2 & \\
\hline Chrysomya megacephala & 19 & 21 & 53 & 47 & 37 & 43 & 183 & 38 & 31 & 34 & 34 & 137 & 29 & 39 & 39 & 107 \\
\hline Chrysomya rufifacies & 0 & 4 & 9 & 7 & 1 & 4 & 25 & 7 & 3 & 2 & 7 & 19 & 5 & 1 & 3 & 9 \\
\hline Lucilia eximia & 2 & 1 & 11 & 5 & 5 & 7 & 31 & 3 & 3 & 2 & 0 & 8 & 0 & 6 & 2 & 8 \\
\hline Cochliomyia macellaria & 0 & 0 & 0 & 0 & 0 & 0 & 0 & 0 & 0 & 0 & 0 & 0 & 1 & 0 & 0 & 1 \\
\hline \multicolumn{17}{|l|}{ Sarcophagidae } \\
\hline Sp1 & 0 & 0 & 0 & 0 & 0 & 0 & 0 & 0 & 0 & 0 & 0 & 0 & 0 & 0 & 0 & 0 \\
\hline Sp2 & 0 & 0 & 0 & 0 & 0 & 0 & 0 & 0 & 0 & 0 & 0 & 0 & 0 & 0 & 0 & 0 \\
\hline Sp3 & 0 & 0 & 0 & 0 & 0 & 0 & 0 & 0 & 0 & 0 & 0 & 0 & 0 & 0 & 0 & 0 \\
\hline \multicolumn{17}{|l|}{ Muscidae } \\
\hline Ophyra Sp & 1 & 1 & 1 & 1 & 4 & 1 & 9 & 1 & 1 & 2 & 0 & 4 & 1 & 0 & 1 & 2 \\
\hline Musca domestica & 0 & 0 & 0 & 1 & 0 & 0 & 1 & 0 & 0 & 1 & 0 & 1 & 0 & 1 & 0 & 1 \\
\hline Sp1 & 0 & 1 & 0 & 0 & 1 & 1 & 3 & 0 & 0 & 0 & 0 & 0 & 0 & 0 & 0 & 1 \\
\hline Sp2 & 1 & 4 & 5 & 0 & 3 & 3 & 16 & 1 & 3 & 4 & 0 & 8 & 3 & 1 & 4 & 8 \\
\hline Sp3 & 0 & 0 & 0 & 0 & 0 & 0 & 0 & 0 & 0 & 0 & & 0 & 0 & 0 & 0 & 0 \\
\hline TOTALES & 26 & 32 & 84 & 62 & 52 & 60 & 276 & 50 & 42 & 46 & 41 & 179 & 40 & 50 & 51 & 137 \\
\hline
\end{tabular}

\section{DISCUSIÓN}

Desde que un ser vivo muere, se inicia el proceso de descomposición que es acompañado por una serie de cambios físicos y químicos que conllevan la liberación de gases, y con ello la liberación de muchas sustancias volátiles que se producen durante los diferentes estados de la descomposición (Barton, 1960). Estas sustancias atraen selectivamente a las moscas, haciendo que acudan cuando la liberación de estas sustancias coincide con su facultad de asimilación nutritiva, en este sentido, la concentración de determinadas sustancias volátiles puede incidir para determinadas especies acudan o no a un cadáver en descomposición, para ovopositor, alimentarse del mismo y reproducirse (Barton, 1960).

La entomología forense puede ayudar a estimar el tiempo transcurrido

Tecnociencia, Vol. 23, $N^{\circ} 1$ 
desde la muerte, estudiando la sucesión de las especies necrófagas recogidas sobre el cadáver. Siendo así, la determinación del IPM se basa en el patrón cronológico de la sucesión de insectos en el cadáver a lo largo del proceso de descomposición (Early y Goff, 1986). Esta estimación se basa principalmente en la afirmación de que las primeras oviposiciones de los insectos necrófagos en el cadáver ocurren en un período cercano a la muerte. Por lo tanto, el conocimiento de los patrones sucesionales puede ayudar en la estimación del IPM.

Dentro de las principales familias que acuden al cadáver o a una carcasa están las familias Calliphoridae, Sarcophagidae y Muscidae, son los primeros insectos que localizan, exploran y colonizan un cadáver a los pocos minutos de ocurrida la muerte (Aspoas, 1994; Tantawi, El-Kady, Greenberg y El-Ghaffar, 1996; Watson y Carlton, 2003; Kyerematen, Boateng y Twumasi. 2012).

En nuestro estudio encontramos que estas familias fueron las más comunes arribando a los hígados en descomposición.

Aunque se observó un claro dominio de las Calliphoridae sobre las Sarcophagidae y la Muscidae. La secuencia de la colonización de artrópodos en cadáveres a nivel de familia es bastante constante en las localidades (Early y Goff, 1986). Sin embargo, a nivel de género y especie, la colonización es específica por la ubicación y se basa en la región zoogeográfica (Early y Goff, 1986). Por lo que, los patrones de sucesión nivel de géneros y especie solo son predecibles dentro de los 
parámetros del lugar donde se encuentra cadáver (Anderson, 2001; Rodríguez y Bass, 1983).

De allí que, en nuestro estudio, cada hígado evidenció una fuerte atracción hacia una especie de mosca en particular, lo que permitió identificar a las que pudieran servir como indicadoras del tiempo de descomposición de los hígados.

Desde el momento en que se colocaron los hígados en el área de estudio, se observó el temprano arribo de las especies Chrysomya megacephala y Lucilia eximia, seguidas de Lucilia sericata, Chrysomya rufifacies y Lucilia cuprina, en los diferentes tejidos en mayor o menor cantidad.

Nuestros resultados coinciden en parte con lo reportado por (Rodríguez y Salazar, 2014) en Venezuela y en Brasil por (Serbino y Godoy, 2017) quienes estudiaron la sucesión en vísceras de res y reportaron la mayor captura de las especies Chrysomya megacephala, Lucilia cuprina, Chrysomya albiceps y Cochliomyia macellaria.

En el hígado expuesto a las 12 horas, la mayor captura correspondió a la especie Lucilia sericata durante los 10 primeros minutos de exposición, la cual exploro el hígado y se alimentó del exudado del mismo. En la segunda hora, también se observó un ejemplar de Lucilia eximia ovipositando sobre el hígado. Esta rápida acción de localizar y colonizar un tejido fresco, a pocas dos horas de haber sido expuestos, la identifican como una buena indicadora del (IPM). También, Vergara- 
Pineda, De León Muzquiz, García-Martínez, Sifuentes-Cantú, Badii y Tomberlin (2009), observaron que Lucilia sericata fue la primero en arribar, a los 15 minutos de haber colocado un cuerpo humano. En Bogotá, Colombia y en Buenos Aires esta especie es considerada eusinantropica, frecuente en áreas urbanas (Centeno, Almorza y Arnillas, 2004; Perez, Duque y Wolff, 2005; Beltran, Segura y Bello, 2012).

Se puedo observar que por las moscas mostraron ciertas preferencias por los diferentes hígados expuestos, Lucilia sericata, en el hígado de 12 y 24 horas; Chrysomya rufifacies en el hígado de 48 horas; Lucilia cuprina y Chrysomya rufifacies en el hígado de 72 horas; Chrysomya megacephala y Chrysomya rufifacies en el hígado de 96 horas de exposición. De acuerdo con Vergara-Pineda, De León Muzquiz, GarcíaMartínez, Sifuentes-Cantú, Badii y Tomberlin (2009), el temprano arribó de Lucilia sericata le permite colonizar los sitios más adecuados del cadáver para el desarrollo de su progenie. El pionerismo de las moscas por encontrar un cadáver fresco, depende de la habilidad diferencial de cada especie de percibir el nitrógeno y sulfuro proveniente de las proteínas degradas (Denno y Cothran, 1975).

En el hígado expuesto a las 48 horas, la mayor captura correspondió a la especie Chrysomya rufifacies. La actividad de esta mosca fue registrada tan pronto se colocó el hígado en el sitio de estudio. VergaraPineda, De León Muzquiz, García-Martínez, Sifuentes-Cantú, Badii y Tomberlin (2009), al igual que Kyerematen. Boateng, Haruna y Eziah, 
(2013) reportaron el arribo temprano de esta especie a los 30 minutos de exposición. Vanegas (2007), considerada esta especie una invasora secundaria en las carcasas.

En el hígado expuesto a las 72 horas de descomposición se encontró que la especie Lucilia cuprina, fue la más frecuente en este tejido, seguido de la especie Chrysomya rufifacies y por último la especie Lucilia sericata. Lo interesante en este hígado fue la gran cantidad de ejemplares de Lucilia cuprina que fueron atraídos, lo que no se observó en ningún otro hígado, lo que sugiere su predilección por tejidos en descomposición en estados avanzados. El potencial forense de esta especie ha sido reportado por Andrade, Varela- Freire, Batista y Medeiros (2005), quienes la reportaron en cinco cadáveres humanos junto a otras cinco especies de Calliphoridae. Barton (1960), reportó que las hembras de esta especie son estimuladas a ovipositar por la presencia de otras moscas.

En el hígado expuesto a las 96 horas, se encontró que la mayor captura correspondió a la especie Chrysomya megacephala, (Fabricius 1794), seguido de la especie Chrysomya rufifacies y de Lucilia eximia. Nuestros resultados coinciden con lo reportado por (Garcés y Rosas, 2016) quienes encontraron las mismas especies en otra área urbana de Panamá. En nuestro estudio la especie Chrysomya megacephala fue registrada en grandes cantidades en el hígado con 96 horas de exposición. La mayor captura de estos especímenes se realizó en la primera hora, de exposición, con 276 especímenes, seguido de la 
segunda hora con 179 especímenes, y, por último, la tercera hora con 137 especímenes (Cuadro 5). Este elevado número de moscas pudiera deberse a que el hígado de 96 horas está más descompuesto y por ende libera mayor cantidad de sustancias volátiles y al tamaño de sus poblaciones naturales.

El arribo masivo de esta especie puede ser su estrategia por acaparar los sustratos pequeños o efímeros. Se registró la captura de sus ejemplares a los 10 minutos de haber colocado el hígado en el sitio de estudio. En ambiente urbanos, algunos autores han reportado la fuerte preferencia de Chysomya megacephala por asentamientos urbanos densos, seguido de Cochliomyia macellaria y Lucilia eximia (Greenberg, 1971).

Por otro lado, Moura, Carvalho \& Monteiro-Filho (1997), reportan que la especie Lucilia eximia puede ser usada como bioindicadora en la estimación del IPM. En el Norte de Río Grande, en Rio de Janeiro, Lucilia eximia ha sido asociada a cadáveres humanos (Salviano, R.J, Mello, Santos, Beck y Ferreira, 1996; Oliveira-Costa, Mello-patiu \& Lopes, 2001; Andrade, Varela- Freire, Batista \& Medeiros, 2005).

Los Sarcophagidae son moscas necrófagas muy importantes, debido a que frecuentemente son encontradas en cadáveres humanos (Goff, 1991; Anderson, 1995; Oliva, 1997). Sin embargo, esta familia ha recibido muy poca atención en la estimación del IPM, debido a la dificultad para identificar a las especies. En la mayoría de los estudios, los ejemplares de Sarcophagidae han sido identificados a nivel de familia y sólo en 
algunos casos hasta género (Wolff, Uribe, Ortiz \& Duque, 2001; Battán, Arnaldos, Rosso \& García, 2005; Aballay, Murúa, Acosta \& Centeno, 2008). En nuestro estudio reconocimos tres morfoespecies, las que reconocemos como $\mathrm{Sp} 1, \mathrm{Sp} 2$ y $\mathrm{Sp} 3$ que arribaron a todos los hígados expuestos, en bajas cantidades. Observamos que una de estas morfoespecies Sp1 larvipositó sobre el hígado de las 96 horas, en la segunda hora de exposición. Las larvas rápidamente se desprendieron su cutícula y penetraron el tejido blando.

Las áreas urbanas han recibido especial atención por la frecuencia con que se registran los homicidios en las ciudades. En la mayoría de los casos, los cuerpos las áreas urbanas se encuentran infestados con los estadios de las moscas. En otros países, en las áreas urbanas, la mayoría de las evidencias entomológicas involucran a las especies Chrysomya albiceps, Chrysomya megacephala, Chrysomya putoria y Lucilia eximia para estimar el IPM (Vasconcelos y Araujo, 2012). Por lo que, es importante en nuestro país realizar los estudios en ambiente antropizados, donde con frecuencia aparecen cadáveres en estado de descomposición avanzado y colonizados por moscas.

La enorme variedad de sustratos que ofrecen los ambientes urbanos, permite que las especies sinantrópicas dominen estos espacios, donde existen o se crean abundantes sustratos efímeros como recursos nutricionales. Otras condiciones que favorecen la presencia de estas moscas necrófagas en las áreas urbanas, son las pobres condiciones higiénicas que facilitan la realización su ciclo biológico, en la basura y 
excretas humanas; el extenso espacio libre que facilita el esparcimiento de las sustancias volátiles y aumenta el forrajeo de los mocas, que son fuertemente atraídas por la gran concentración de las sustancias volátiles.

\section{CONCLUSIONES}

La especie Lucilia sericata, fue la primera arribo a los primeros minutos de haber expuesto los hígados de 12 y 48 horas y, colonizo el mismo depositando una masa de huevos, en la segunda hora de exposición.

Las principales especies que evidenciaron cierta preferencia por los diversos hígados expuestos, en los diferentes intervalos de tiempo, fueron Lucilia sericata, Lucilia cuprina, Chrysomya rufifacies y Chrysomya megacephala.

Las cuatro especies pudieran ser consideradas como buenas indicadoras forenses en los ambientes urbanos, debido a que rápidamente arriban a cadáveres humanos o de animales o de hígados humanos expuestos.

La especie Lucilia cuprina fue registrada en forma constante en el hígado de 72 horas de exposición, lo que sugiere que puede ser una colonizadora de estados avanzados de descomposición.

La mayor captura de la especie Chrysomya megacepha ocurrió en el hígado de 96 horas, lo que sugiere que tan pronto siente un fuerte olor fétido acuden en grandes cantidades a dominar la colonización, a alimentarse de los exhudados o a ovipositar. 


\section{REFERENCIAS}

Aballay, F.H., Murúa, A.F, Acosta, J.C. y Centeno, N.D. (2008). Primer registro de artropodofauna cadavérica en sustratos humanos y animales en San Juan, Argentina. Revista de la Sociedad Entomológica Argentina, 67: 157-163.

Amat, E.V., Ramíre-Mora, M.A., Buenaventura, E. y Góme-pinerez, L.M. (2013). variación temporal de la abundancia en familias de moscas carroñeras (diptera, calyptratae) en un valle andino antropizado de colombia. Acta Zoológica Mexicana. 29: 463-472.

Amat, E.V. y Wolff, M. (2008). Clave ilustrada para la identificación de los géneros y las especies de califóridos (Diptera: Calliphoridae) de Colombia. Caldasia. 30:231-244.

Anderson, G.S. (2001). Insect succession on carrion and its relationship to determiningthe time of the death. En: Byrd J.H. y J.L. Castner, (eds.). Forensic Entomol. The Utility of Arthropods in Legal Investigations. CRC Press LLC. U.S.A. 418 p. 143-17.

Anderson, G.S. y VanLaerhoven, S.L. (1996). Initial studies on insect succession on carrion in southwestern British Columbia. Journal of Forensic Sciences \& Criminal Investigation, 41: 617-625.

Anderson, G.S. (1995). The use of insects in death investigations: an analysis of cases in British Columbia over a five-year period. Canadian Society of Forensic Science Journal, 28: 277-292. 
Andrade, H.T., Varela- Freire, A.A, Batista M.J. y Medeiros, J.F. (2005). Calliphoridae (Diptera) Coletados em Cadáveres Humanos no Rio Grande do Norte. Neotropical Entomology. 34:855-856.

Aspoas, B.R. (1994). Afrotropical Sarcophagidae in a carrion fly community. Medical and Veterinary Entomology. 8:292-294.

Barbosa, R.R., Mello-Patiu, C.A, Ururahy-Rodrigues, A, Guimarães Barbosa, C., y Carvalho Queiroz, M. M. (2010). Temporal distribution of ten calyptrate dipteran species of medicolegal importance in Rio de Janeiro, Brazil. Mem Inst Oswaldo Cruz. 105: 191-198.

Barton, B.L. (1960). The role of olfaction in the stimulation of oviposition in the blowfly, Phormia Regina. Journal of Insect Physiology. 5: 16-22.

Battán, H.M., Arnaldos, M.I, Rosso, B. y García, M.D. (2005). Estudio preliminar de la comunidad sarcosaprófaga en Córdoba (Argentina): aplicación a la entomología forense. Anales de Biología. 27: 191-201.

Beltran, Y.T., Segura, N.A. y Bello, F.J. (2012). Synanthropy of Calliphoridae and Sarcophagidae (Diptera) in Bogota, Colombia. Neotropical Entomologia. 41: 237-242.

Byrd, L. y Castner, J.L. (2001). Forensic Entomology: the Utility of 
Arthropod in Legal Investigations. CRC Press LLC. U.S.A. 418 p.

Camacho, G. (2003). Sucesión de la entomofauna cadavérica y ciclo vital de Calliphora vicina (Diptera: Calliphoridae), como primera especie colonizadora, utilizando cerdo blanco (Sus scrofa) en Bogotá. Revista Colombiana de Entomología, 31:189-127.

Carvalho, L.M., Thyssen, P.J., Goff, M.L. y Linhares, A.X. (2004). Observations on the succession patterns of necrophagous insects on a pig carcass in a urban area of Southeastern Brazil Aggrawal's Internet J. Forensic Med. Toxicol. 5: 33-39.

Catts, E.P. y Haskell, N.H. 1997. Entomology and Death: A Procedural Guide. Joyce's Print Shop. Clemson, South Carolina, 183 pp

Catts, E.P. y Goff, M.L. (1992). Forensic entomology in criminal investigations. Annual Review of Entomology, 37: 253-272.

Centeno, N., Almorza, D. y Arnillas, C. (2004). Diversity of Calliphoridae (Insecta: Diptera) in Hudson, Argentina. Neotropical Entomology, 33: 387-390.

Denno, R.F. \& Cothran, W.R. (1975). Niche relationships of a guild of necrophagous flies. Annals of the Entomogical Society of America. 68: 741-754.

Early, M. y Goff, M.L. (1986). Arthropod succession patterns in exposed carrion on the island of O'ahu, Hawaiian Islands, USA. Journal Tecnociencia, Vol. 23, $N^{\circ} 1$ 
of Medical Entomology. 23: 520-531.

Garcés, P.A. y Rosas, M. (2016). Comparación de las poblaciones de moscas necrófagas en dos localidades de Panamá y Chiriquí. Revista científica CENTROS.5: 56-88.

Goff, M.L. (1991). Comparison of insect species associated with decomposing remains recovered inside dwellings and outdoors on the Island of Oahu, Hawaii. Journal of Forensic Sciences, 36: 748-753.

Greenberg, B. (1971). Flies and disease Ecology, classification and biotic association. Princeton University Press, New Jersey

Kyerematen, R.A., B.A Boateng y E. Twumasi. (2012). Insect diversity and succession pattern on different carrion types. Journal of Research in Biology, 2: 683-690.

Kyerematen, R.A., Boateng, B.A., Haruna, M. y Eziah, V. Y. (2013). decomposition and insect succession pattern of exposed domestic pig (sus scrofa 1.) carrion. International Journal of Agriculture and Biological Sciences, 8: 756-765.

Moura, M.O., Carvalho, C.J. y Monteiro-Filho, E.L. (1997). A preliminary analysis of insects of médico-legal importance in Curitiba, State of Paraná. Memorias del Instituto Oswaldo Cruz, 92: 269-274.

Oliva, A. (1997). Insectos de interés forense de Buenos Aires (Argentina). Primera lista ilustrada y datos bionómicos. Revista del 
Museo Argentino de Ciencias Naturales, 7: 13-59.

Oliveira-Costa, J., Mello-patiu, C.A., y Lopes, S.M. 2001. Dípteros muscóides associados com cadáveres humanos na cena da morte no estado do Rio de Janeiro, Brasil. Boletim do Museu Nacional, Zoologia. 1564: $1-6$.

Perez, S.P., Duque, P. y Wolff, M. (2005). Successional behavior and occurrence matrix of carrion-associated arthropods in the urban area of Medellin, Colombia. Journal of Forensic Sciences, 50: 1-7.

Ramos-Pastrana, Y., Velasquez-Valencia, A. y Wolff, M. (2014). Preliminary study of insects associated to indoor body decay in Colombia. Revista Brasileira de Entomología. 58: 326-332

Rodríguez, J.N. y Salazar, J.L. (2014). Sucesión de la entomofauna cadavérica a partir de un biomodelo con vísceras de res. Revista de la Facultad de Ciencias de la Salud. Universidad de Carabobo. 18: 35-39.

Rodriguez, W.C y Bass, W.M. (1983). Insect activity and its relationship to decay rates of human cadavers in east Tennessee. Journal of Forensic Sciences, 28:423-432.

Sanabria, M.C. (2008). Antropología forense y la investigación médico legal de las muertes. 2da edición. Asociación Colombiana de antropología forense. 
Salviano, R.J., Mello, R.P., Santos, R.F., Beck, L.C. y Ferreira, A. (1996). Calliphoridae (Diptera) associated with human corpses in Rio de Janeiro, Brazil. Entomologia y Vectores. 3: 145-146.

Schoenly, K.G., Hankell, N.H., Mills, D.K., Bieme, C., Larsen K., y Lee, Y. (2006). Using pig carcasses as model Corpes. The American Biology Teacher. 68: 402-409

Smith, K.G. (1986). A manual of forensic entomology. The trustees of the British Museum (Natural history) and Cornell University Press, New York. 205 p.

Tantawi, T., El-Kady, E.M, Greenberg, B. y El-Ghaffar, H.A. (1996). Arthropod succession on exposed rabbit carrion in Alexandria, Egypt. Journal of Medical Entomology. 33: 566-580.

Tomberlin, J.K., Benbow, M. E, Tarone, A.M. y Mohr, R.M. (2011). Basic research in evolution and ecology enhances forensics. Trends in Ecology \& Evolution, 2653-55.

Vanegas, S.Y. (2007). Efectos de la temperatura sobre el desarrollo de Chrysomya rufifacies y Cochliomyia macellaria (Diptera: Calliphoridae), dos especies importantes para la entomología forense en Puerto Rico. Tesis Universidad de Puerto Rico. 98pp.

Vasconcelos, S.D. y Araujo, M.C. (2012). Necrophagous species of Diptera and Coleoptera in northeastern Brazil: state of the art and challenges for the Forensic Entomologist. Revista Brasileira de Garcés, P.A \& Colaboradores 48 
Entomologia, http://dx.doi.org/10.1590/S0085-56262012005000014

Vergara-Pineda, S., De León Muzquiz, M.H., García-Martínez, O., Sifuentes-Cantú, M., Badii, M.H. y Tomberlin, J.K. 2009. Comportamiento de arribo de Moscas necrofagas (Diptera: Calliphoridae) a un Cadáver Humano. Entomología Mexicana. 8: 792797.

Von Zuben, C.J., Bassanezi, R.C, Reis, S.F, Godoy, W.A. y Zuben, F.J. 1996. Theoretical approaches to forensic entomology: I. Mathematical model of postfeeding larval dispersal. Journal of Applied Entomology, 120: $379-382$.

Watson E. y Carlton, C. (2003). Spring succession of necrophilous insects on wildlife carcasses in Louisiana. Journal of Medical Entomology, 40: 338-347.

Wolff, M., Uribe, A, Ortiz, A. y Duque, P. (2001). A preliminary study of forensic entomology in Medellín, Colombia Forensic Science International Journal, 120: 53-59.

Recibido 15 abril y aceptado 16 noviembre 2020

Tecnociencia, Vol. 23, $N^{\circ} 1$ 